\title{
Selecting and Screening Donors
}

\author{
Sahadat Kemi Nurudeen, Brian A. Levine, \\ and Melvin $\mathrm{H}$. Thornton II
}

\section{Key Points}

- Screening of egg donors is an intricate and multifaceted process that includes obtaining informed consent; securing a detailed medical, genetic, psychosocial, and reproductive history; performing a thorough physical examination; and testing for specific infectious diseases.

- Screening criteria proposed by ASRM recommends that all donors should be in excellent health and without history of hereditary or communicable diseases.

S.K. Nurudeen, M.D.

Department of Obstetrics and Gynecology, College of Physicians and Surgeons, Columbia University,

New York Presbyterian Hospital-Columbia University Medical Center, 622 West 168th Street, 16th Fl,

Rm 64 West, New York, NY 10032, USA

e-mail: sknurudeen@gmail.com

B.A. Levine, M.D., M.S.

Department of Obstetrics and Gynecology, College of Physicians and Surgeons, Columbia University,

New York Presbyterian Hospital-Columbia University

Medical Center, 622 West 168th Street, PH-16,

New York, NY 10032, USA

e-mail: brian.a.levine@gmail.com,

bal2138@columbia.edu

M.H. Thornton II, M.D. $(\bowtie)$

Division of Reproductive Endocrinology and Infertility, Department of Obstetrics and Gynecology, College of Physicians and Surgeons, Columbia University,

New York Presbyterian Hospital-Columbia University

Medical Center, 622 West 168th Street, PH-16,

New York, NY 10032, USA

e-mail:mt760@columbia.edu
- The FDA requires a full historical assessment of potential infectious disease risk factors as well as the performance of specific tests utilizing nucleic acid testing (NAT) for HIV and hepatitis C within 30 days of egg harvest.

- Anti-Müllerian hormone (AMH) represents an accurate serum marker for ovarian responsiveness to ovarian stimulation and is a useful adjunctive measure in predicting both poor response and hyperresponse in oocyte donors.

Oocyte donation was originally established in 1983 as a treatment option for younger women with premature ovarian failure and for women with severe pelvic disease whose ovaries, as a result, were surgically inaccessible $[1,2]$. The indications for donor oocyte in vitro fertilization (IVF) have now expanded to include not only women with hypergonadotropic hypogonadism but also those with advanced reproductive age, diminished ovarian reserve, significant genetic disease risk, poor oocyte or embryo quality, or multiple failures in prior attempts to conceive using conventional assisted reproductive technology (ART). Oocyte donation has also been recently used as an important source of material to promote the study of stem cell research [3].

In these first cases of donation, gametes were obtained primarily from women already undergoing 
Table 4.1 Summary of institutional guidelines for laboratory testing of oocyte donors

\begin{tabular}{llll}
\hline & FDA [8] & ASRM [7] & NYSDH $^{\mathrm{a}}$ \\
\hline ABO and Rh type & & $\mathrm{X}$ & $\mathrm{X}$ \\
\hline HIV-1 and HIV-2 & $\mathrm{X}$ & $\mathrm{X}$ & $\mathrm{X}$ \\
\hline Hepatitis B core antibody & $\mathrm{X}$ & $\mathrm{X}$ & \\
\hline Hepatitis B surface antigen & $\mathrm{X}$ & $\mathrm{X}$ & $\mathrm{X}$ \\
\hline Hepatitis C antibody & $\mathrm{X}$ & $\mathrm{X}$ & $\mathrm{X}$ \\
\hline Human T-lymphotropic virus (type 1) & & & $\mathrm{X}$ \\
\hline Syphilis & $\mathrm{X}$ & $\mathrm{X}$ & $\mathrm{X}$ \\
Chlamydia & $\mathrm{X}$ & $\mathrm{X}$ & $\mathrm{X}$ \\
\hline Gonorrhea & $\mathrm{X}$ & $\mathrm{X}$ & $\mathrm{X}$ \\
\hline West Nile virus & & $\mathrm{X}$ &
\end{tabular}

FDA US Food and Drug Administration, ASRM American Society for Reproductive Medicine, NYSDH New York State Department of Health

a Reproductive Tissue Banks. Part 52 of Title 10 (health) of the official compilation of codes, rules and regulations of the state of New York 2007; Subpart 52-8:64-74
IVF who had excess oocytes at the time of retrieval [1]. Today, most egg donors are not currently pursing infertility treatment themselves but are willing to donate their gametes for altruistic or commercial reasons. Since its initiation, oocyte donation services have spread throughout the USA and to many areas of the world. In the USA, 9,000-10,000 donor oocyte cycles occur annually [4]. Though donor oocyte IVF is available throughout the USA, globally the practice of oocyte donation varies due to legal restrictions in many countries (Chap. 30).

The compensation and recruitment practices for oocyte donors vary worldwide and largely depend upon current legal or cultural practices in that locale. For instance, countries, such as the UK and Canada, have strict restrictions on donor compensation, while others, such as Italy and Germany, prohibit compensation [5]. Donor compensation guidelines do not exist in the USA, and regional differences in compensation do occur $[6,7]$. In the USA, donors are recruited mainly through the Internet, television, radio, and newspaper advertisements. Donors are "matched" to recipient couples often based on educational credentials, extracurricular activities, phenotypic traits, and ethnic origins. Though providing recipients with the choice of egg donors who exhibit these traits and qualities is important, the selection and screening process is much more comprehensive in order to ensure the safety and health of the donor, recipient, and the offspring.

\section{Screening Oocyte Donors}

Screening women interested in becoming oocyte donors is an intricate and multifaceted process that includes obtaining informed consent, taking a thorough medical history, performing a complete medical examination, testing for infectious diseases, providing a genetic screen, and evaluating the donor psychologically. Ideally, programs want to secure the services of women who are in good health without any past history of risky behavior or familial diseases. The screening process has evolved since the introduction of oocyte donation with recommendations and evidence provided by the American Society for Reproductive Medicine (ASRM), the US Food and Drug Administration (FDA), Centers for Disease Control and Prevention (CDC), and state health departments (Table 4.1) [8,9]. The FDA finalized the donor eligibility and the good tissue practice rules to ensure public safety through proper screening for risk factors and testing of donors for pertinent transmissible diseases [9].

Before putting potential oocyte donors through the screening process, it should be determined whether they meet the initial requirements for donor selection. In general, donors should be healthy without a history of hereditary disease. Donors are recommended to be between ages 21 and 34 years of age according to the 2008 ASRM screening guidelines [8]. Using donors less than 21 years of age should be determined on an individual basis 
after a thorough psychological evaluation by a qualified mental health professional [8]. The use of donors over age 34 requires a discussion of the risk of aneuploidy and lower pregnancy rates associated with older women [10-12]. Those individuals with employment ties to or financial interests in a donor oocyte program or recruiting agency should not be used as oocyte donors due to the obvious conflict of interest.

\section{Informed Consent}

Prior to participating in oocyte donation, the potential legal, medical, and psychosocial issues involved in the process should be discussed with the donor while obtaining informed consent. Legal issues include but are not limited to understanding rights to maintain and protect anonymity, discovering outcomes of donation, and whether or not future contact with any resulting children is desired [13]. For example, future anonymity could theoretically be reversed in court situations where the importance of the offspring knowing his/her genetic background is deemed to outweigh the donor's desire for privacy [13]. Arguments in favor of revealing the donor's identity stem from the fact that children born through donated oocytes were not a part of the original decision-making process. Though not yet regularly employed by centers nationally, informed consent should also contain information about the disposition of cryopreserved embryos resulting from oocyte donation, i.e., use for future pregnancies or donation for research [3, 13, 14]. However, these cryopreserved embryos cannot be reclaimed by donors in the future [13]. In summary, the potential donor should consider all legal matters both known and possible during the informed consent process (further discussed under Obligations and Rights).

Detailed medical risks involved with the process of oocyte donation should also be discussed while consenting. Donors need to be aware of the acute risks and adverse effects of ovarian stimulation and oocyte retrieval, including but not limited to ovarian hyperstimulation syndrome (OHSS), intraperitoneal hemorrhage, and pelvic infection (Chap. 19) [15]. There may also be risks associated with the use of anesthesia provided during the oocyte retrieval including allergic reaction to anesthetic drugs and respiratory compromise secondary to aspiration. However, among the young healthy population of donors, this risk is very small [16]. In fact, previous studies have suggested that donors are at lower risk for OHSS and other adverse events than patients undergoing autologous IVF [17]. Programs may wish to provide donors with adequate supplemental insurance coverage for medical complications arising from oocyte donation, and the terms of such coverage should be disclosed to the donor (further discussed in Chap. 20).

The potential increased risk of ovarian cancer should also be introduced during the discussion of the possible medical risks from oocyte donation. There have been concerns that controlled ovarian stimulation (COS) might increase the long-term risk of ovarian cancer in women using fertility drugs. Recently published data have not confirmed a cause and effect relationship between the drugs used for COS and ovarian cancer [18]. However, undoubtedly most donors have either heard about the association of fertility drugs and cancer or read that it exists in the popular media or Internet, and therefore, questions regarding the long-term safety of participation should be anticipated.

Unintended pregnancy in donors discontinuing oral contraceptives in order to participate also occurs. Potential donors should be counseled on this possibility and offered options for prevention [8]. Along with the discussion of pregnancy risk during informed consent, it is important to stress the importance of patient compliance with followup, use of contraception, and/or abstinence in order to lessen the risk of unplanned pregnancy.

Egg donors should be advised about the emotional and psychosocial consequences of participation. They should understand the potential impact that providing eggs to an infertile woman may have upon their own offspring or future offspring, including whether or not to ever disclose to their own children or spouse the fact that they formerly participated. Although highly unlikely, there are notable concerns that offspring could 
potentially marry and procreate with an unrecognized half-sibling [16]. In addition, the donor's present or future spouse or partner may have an interest in the outcome of prior oocyte donation, and disclosure may have a negative effect on their relationship [13].

\section{Defining Obligations, Rights, and Duties}

Obligations, rights, and duties of donors should be thoroughly explained prior to participation. For instance, donors may ask about the right to specify the type of recipients that receive their donation, the right to learn about the outcome of their donations, the right to contact any future offspring, and, as discussed previously, their right to anonymity. Each of these obligations must be agreed upon prior to initiating a cycle of treatment.

Entering the oocyte donation process, some donors believe that they may direct their egg donation to include only specific demographics of recipients. Such triage might be based on the age of the recipients, or perhaps their marital status, sexual orientation, health, race, religion, or education. The request of donors to specify to whom their gametes may be given is typically refused by donor programs, except in the obvious cases of designated friends or family members openly participating with known recipients. The choice to not allow anonymous donors to direct the use of their gametes is currently supported by the Ethics Committee of the ASRM [13]. Potential donors should understand that their preferences to donate only to certain types of recipients will likely not be considered in gamete donation. Furthermore, the future use of embryos created by the donated eggs lies with the recipient and cannot be easily predicted or later controlled.

Programs should give consideration to the fact that donors may express an interest in learning the outcome of their donation. Whether or not outcomes will be disclosed should be defined prior to participation. According to current guidelines, programs are not ethically bound to reveal whether or not a pregnancy occurred because the donation is made without regard to the outcome which is consistent with current blood and non-gamete tissue donation practices [13]. Disclosing information about cycle success or failure may at times cause unanticipated emotional distress to donors possibly secondary to the news of offspring or, in cases of failure, cause concern about the donor's own fertility [13]. Some argue that outcomes should be disclosed because donors deserve to know whether their gametes resulted in a successful pregnancy. The knowledge of outcomes could be helpful in the event of planned or unplanned contact from the offspring, give donors the opportunity to tell their children about genetic half-siblings, and put psychological closure on their participation in oocyte donation [13]. Some programs allow donors the option of learning whether a child was born, yet there exist few research studies to support disclosure or nondisclosure of pregnancy to determine which approach is preferable.

We believe that donors should be asked and documented as to whether they are willing to have contact with any offspring. Initially, some participants may be content with simply providing their gametes. However, in the future, some donors may have an interest in knowing their offspring. On the other hand, donors have the right of not having potential obligations to offspring imposed on them without their consent [13]. These are strong considerations, and asking them to anticipate their future inclination is complex given that their feelings may and are likely to change considerably during their lifetime.

There may be competing interests between donors, recipients, and subsequent offspring. Disclosing to offspring the donor's genetic history does not necessarily require knowing the actual identity of the donor or meeting her. However, with increasing interest in the issues surrounding future contact between donors and their offspring, there should be some acknowledgement of the potential for new situations and responsibilities to arise concerning participants as regulations and laws change in the future. It has also been suggested that donors and recipient couples may wish to consider executing legal documents that attempt to define or limit the rights and duties of each with regard to any future offspring [13]. 
The need for compliance with treatment should be stressed to oocyte donors. This will assist in maximizing cycle outcomes and minimizing the potential medical risks. Informed consent requires donors to be forthcoming about their personal medical history and behaviors so that any genetic and/or health issues that affect the well-being of offspring are known in advance [13]. It is the responsibility of the donor to update the donor program with any changes to her health or risk factor status [8]. However, it is less clear about the donor's obligations after donation to keep the program informed of any changes in her health status. Programs may encourage donors to provide updates as they encounter medical conditions that may be pertinent to the offspring's health. Standard operating procedures (SOP) should be in place with regard to medical updates, and programs should clearly convey this to their participating donors and the recipients of donor eggs.

Donors should be assured that their confidentiality will be protected as federal and local state laws permit. The medical records containing the information about their participation will be protected and sustained according to local statutes [8]. The FDA requires that the records of donor screening and testing be maintained for at least 10 years; ASRM actually recommends maintaining a permanent record of each donor's selection process, screening, testing, and follow-up evaluations [8]. These records, including those with clinical outcomes, should be maintained for any potential information sharing in the future with offspring based on future statutes or permission of the donor.

\section{Past Medical History}

When evaluating a potential oocyte donor, a comprehensive review of their past medical history is requisite. According to the recommended screening criteria of the ASRM, the donor should be healthy and give no history to suggest hereditary or communicable disease [8]. The goals of screening are to ensure that the donor is not at risk for suffering an untoward event during the stimulation/ retrieval process and to also ensure that the donor is
Table 4.2 FDA/ASRM donor history screening guidelines [7]

\begin{tabular}{|c|}
\hline Medical history \\
\hline History of hereditary disease \\
\hline $\begin{array}{l}\text { History of hemophilia or other coagulation disorders } \\
\text { who have received human-derived clotting factor } \\
\text { concentrates }\end{array}$ \\
\hline $\begin{array}{l}\text { Exposure within the last } 12 \text { months to blood known } \\
\text { or suspected to be infected with HIV, hepatitis B, } \\
\text { and/or hepatitis C virus }\end{array}$ \\
\hline $\begin{array}{l}\text { Treatment for syphilis, gonorrhea, or chlamydia } \\
\text { within last } 12 \text { months }\end{array}$ \\
\hline $\begin{array}{l}\text { Risk for or family history of transmissible spongi- } \\
\text { form encephalopathy }\end{array}$ \\
\hline Recent West Nile viral infection \\
\hline $\begin{array}{l}\text { Acute SARS infection or risk factors for SARS } \\
\text { infection }\end{array}$ \\
\hline $\begin{array}{l}\text { History of xenotransplant or close contact with } \\
\text { xenotransplant patient }\end{array}$ \\
\hline $\begin{array}{l}\text { History of human organ or tissue transplant or } \\
\text { human extracts }\end{array}$ \\
\hline Recent smallpox vaccination \\
\hline Social history \\
\hline $\begin{array}{l}\text { Injected drug use for nonmedical reasons in last } \\
5 \text { years }\end{array}$ \\
\hline $\begin{array}{l}\text { Engagement in casual sexual relations frequently } \\
\text { with different partners }\end{array}$ \\
\hline $\begin{array}{l}\text { Participation in sex in exchange for money or drugs } \\
\text { in the last } 5 \text { years }\end{array}$ \\
\hline $\begin{array}{l}\text { History of incarceration in jail for more than } 72 \mathrm{~h} \text { in } \\
\text { the last } 12 \text { months }\end{array}$ \\
\hline $\begin{array}{l}\text { Living with another person with viral hepatitis } \\
\text { within last } 12 \text { months }\end{array}$ \\
\hline $\begin{array}{l}\text { Acupuncture, body piercing, or tattooing procedures } \\
\text { within the last } 12 \text { months in which sterile procedures } \\
\text { were not or suspected to be not used }\end{array}$ \\
\hline $\begin{array}{l}\text { Sexual intercourse in the last } 12 \text { months with persons } \\
\text { with any of the above medical or social history }\end{array}$ \\
\hline
\end{tabular}

not at risk of transmitting a possible blood-borne pathogen. The ASRM Practice Committee recommendations for screening oocyte donors are not law and are strictly guidelines from the professional organization based on regulation from the FDA. ASRM, in congruence with the FDA, has recommended that donor programs not accept donors, who in the past 5 years, have injected drugs for nonmedical reasons, have received humanderived clotting factor concentrates, or have had sex in exchange for money or drugs (Table 4.2) [8]. Donors, who in the preceding 12 months, have had sex or close contact with any person having HIV 
infection including a positive or reactive test to HIV virus, hepatitis B infection, or clinically active (symptomatic) hepatitis $\mathrm{C}$ infection; have been incarcerated in a lockup, jail, or prison for more than 72 consecutive hours; had or have been treated for syphilis, gonorrhea, or chlamydia; or have undergone body piercing and/or tattooing procedures in which sterile procedures were not used or it is unclear whether sterile procedures were used, should also be excluded (Table 4.2) [8].

The FDA requires the further assessment of potential risk factors based upon the donor's travel history, given that many individuals may be harboring indolent communicable infections. The criteria clearly recommend rejecting women who spent 3 months or more cumulatively in the UK from the beginning of 1980 through the end of 1996; those who are current or former US military members, civilian military employees, or dependents of a military member or civilian employee who resided at US military bases in Northern Europe (Germany, Belgium, and the Netherlands) for 6 months or more cumulatively from 1980 through 1990, or elsewhere in Europe (Greece, Turkey, Spain, Portugal, and Italy) for 6 months or more cumulatively from 1980 through 1996; those who spent 5 years or more cumulatively in Europe from 1980 until present; those who received any transfusion of blood or blood components in the UK or France between 1980 and the present; those whom sexual partners who were born or lived in certain countries in Africa (Cameroon, Central African Republic, Chad, Congo, Equatorial Guinea, Gabon, Niger, or Nigeria) after 1977; and those who have received a blood transfusion or any medical treatment that involved blood in the countries listed above after 1977 [9].

Interestingly, the FDA makes special note about the importance of including a comprehensive review of symptoms to ensure that no donors are included who are at risk for West Nile virus. Given that the disease can have profound neurologic sequelae, those who are at risk or have symptomatology consistent with an infection are recommended to defer donation for at least 120 days after onset of symptoms or diagnosis, whichever is later [9]. Along the same lines of communicable neurologic disease, the FDA bans women from donating who have been diagnosed with variant Creutzfeldt-Jakob disease (vCJD) or any other form of CJD, diagnosed with dementia or any other degenerative or demyelinating disease of the central nervous system or other neurologic disease of unknown etiology, received a non-synthetic dura mater transplant, human pituitary-derived growth hormone, or have one or more blood relatives diagnosed with CJD. Even those women who have a history of CJD in a blood relative should not be included unless the diagnosis of CJD was subsequently found to be in error, the CJD was iatrogenic, or laboratory testing (gene sequencing) demonstrates that the donor does not have a mutation associated with familial CJD [9].

Lastly, given the potential for infection and transmission of pathogens to patients receiving organ transplants, those women who have received xenotransplants (live cells, tissues, or organs from a nonhuman animal source or human body fluids, cells, tissues, or organs that have had ex vivo contact with live nonhuman animal cells, tissues, or organs), have been in close contact with a xenotransplant recipient, or have received human organ or tissue transplants or treatment with human extracts are not eligible as well [9].

Though not listed in the published guidelines, physicians may also consider other aspects the donor's medical history that could preclude or limit participation in egg donation. Knowledge of current medical conditions, such as polycystic ovarian syndrome, may assist clinicians in selecting appropriate treatment protocols in order to reduce the risk for ovarian hyperstimulation syndrome. Donors with a history of obesity, endometriosis, or pelvic surgery will alert clinicians to possible diminished ovarian reserve or difficulty with ovarian access during retrieval. Finally, clinicians may consider assessing the donor's family history for other inheritable traits and diseases, such as color blindness, diabetes, or premature ovarian failure.

\section{Physical Exam}

The screening of oocyte donors must include a thorough and focused physical exam. When performing the physical exam, the ASRM has outlined 
Table 4.3 ASRM recommended components of the oocyte donor's physical exam [7]

Physical evidence for risk of sexually transmitted disease such as genital ulcerative lesions, herpes simplex, chancroid, and urethral discharge

Physical evidence for risk of or evidence of syphilis

Physical evidence of anal intercourse including perianal condylomata

Physical evidence of nonmedical percutaneous drug use such as needle tracks; the examination should include examination of tattoos, which might be covering needle tracks

Physical evidence of recent tattooing, ear piercing, or body piercing

Disseminated lymphadenopathy

Unexplained oral thrush

Blue or purple spots consistent with Kaposi sarcoma

Unexplained jaundice, hepatomegaly, or icterus

Large scab consistent with recent history of smallpox immunization

Eczema vaccinatum, generalized vesicular rash, severely necrotic lesion (consistent with vaccinia necrosum), or corneal scarring (consistent with vaccinial keratitis)

the requisite components (Table 4.3) focused on assessing the donor's general health and potential for harboring an infection that may be transmitted to a recipient. As discussed previously, the donor should also be evaluated for pelvic findings that might complicate the treatment (i.e., polycystic appearing ovaries, endometriosis, and pelvic disease) with a transvaginal ultrasound.

\section{Laboratory Testing}

\section{Infectious Disease}

A thorough medical history, as discussed previously, is important in determining individuals at high risk for infection. The testing for infection among potential donors has been regulated by the FDA and American Association of Tissue Banks (Table 4.4). Testing should be performed within 30 days prior to oocyte collection, and abnormal results need to be verified prior to disclosure to the donor [8]. During disclosure, centers should have options for counseling and medical referral if needed. Any positive screening tests will exclude potential donors from anonymous donation except for a history of treated Neisseria gonorrhoeae and Chlamydia trachomatis infections [8]. Oocyte donation should be deferred for 12 months after a negative test of cure for donors who have a history of these specific infections [8]. However, due to the increased risk of infertility in these women, in general, participation should be discouraged.

In the event that a concern for donor infection arises during a treatment cycle, recipients should be offered the option of embryo cryopreservation and quarantine for 180 days until the donor is tested and confirmed negative for infection [8]. In such rare events, the program should also properly counsel recipients about their frozen embryo transfer (FET) pregnancy rates and the chance of seroconversion of the donor after cryopreservation of embryos in order for the recipients to make appropriate decisions regarding pursuit of FET.

Non-anonymous donors undergo the same testing as anonymous donors. However, known donors who test positive are not automatically excluded from oocyte donation according to the current FDA guidelines as long as the physician is aware of the results [9]. Though the FDA does not require disclosure of positive test results, ASRM recommends informing and properly counseling recipient couples prior to use of oocytes [8].

There are no formal recommendations for testing of the male partners of recipients, but a few tests may be considered. These include semen analysis, blood type, $\mathrm{Rh}$ factor, infectious disease blood work, and genetic screening depending on the male partner's ethnic background [8].

\section{HIV}

Centers should test for the HIV-1 and HIV-2 antibodies with enzyme immunoassay (EIA) techniques 
Table 4.4 FDA-approved infectious disease laboratory tests [8]

\begin{tabular}{|c|c|c|}
\hline & Source & Test \\
\hline \multirow[t]{2}{*}{ HIV } & Serum & EIA HIV-1 and HIV-2 antibodies \\
\hline & & NAT test for HIV-1 \\
\hline \multirow[t]{2}{*}{ Hepatitis B } & Serum & Hepatitis B surface antigen \\
\hline & & EIA hepatitis B core antibodies (IgG and IgM) \\
\hline \multirow[t]{2}{*}{ Hepatitis C } & Serum & EIA hepatitis $\mathrm{C}$ antibody \\
\hline & & NAT for $\mathrm{HCV}$ \\
\hline \multirow[t]{2}{*}{ Syphilis } & Serum & $\begin{array}{l}\text { Nontreponemal (initial): RPR, VDRL, automated } \\
\text { reagin test }\end{array}$ \\
\hline & & $\begin{array}{l}\text { Treponemal (confirmatory): FTA, TP-PA, TPI, } \\
\text { or EIA for specific antibodies to T. pallidum }\end{array}$ \\
\hline \multirow[t]{2}{*}{ Neisseria gonorrhoeae } & Cervix, vagina, and urine & Culture \\
\hline & & NAT \\
\hline \multirow[t]{2}{*}{ Chlamydia Trachomatis } & Cervix, vagina, urethral meatus, & Culture \\
\hline & and urine & NAT \\
\hline
\end{tabular}

EIA enzyme immunoassay, NAT nucleic acid testing, TP-PA T. pallidum particle agglutination, TPI T. pallidum immobilization test

and utilize the nucleic acid testing (NAT) for viral particles. The FDA requires the use of nucleic acid tests because of the ability to detect infection at a significantly earlier stage than traditionally used antibody or antigen testing $[9,19]$. There are tests currently available that are also sensitive for the detection of HIV group $\mathrm{O}$ antibodies. Centers that do not have access to the FDA-licensed test for the group $\mathrm{O}$ antibodies should evaluate donors for risks associated with HIV group O infection [9]. Participants that may be at risk include those who were born, lived in, or received blood transfusion or any medical treatment in Cameroon, Central African Republic, Chad, Congo, Equatorial Guinea, Gabon, Niger, or Nigeria after 1977 [8].

\section{Hepatitis}

Serologic testing for hepatitis B infection utilizes EIA for the detection of antigens and antibodies, specifically the hepatitis B surface antigen and hepatitis B core antibodies (IgG and $\operatorname{IgM})[8,20]$. These tests will reveal any infected donors and further distinguish between acute or chronic hepatitis. Though the majority of adults infected with the virus experience recovery, $1-5 \%$ of immunocompetent adults are at risk for chronic hepatitis and thus cirrhosis and hepatocellular carcinoma [21]. Viral infection acquired during childhood or the perinatal period has a risk of persistent infection ranging from 20 to $90 \%$ [21]. Women infected with hepatitis B infection should be excluded from egg donation and given appropriate recommendations for follow-up with a primary care physician or infectious disease specialist. Many donors may have received hepatitis B vaccination in childhood or adolescence. In these instances, past immunization may be confirmed by testing the serum for hepatitis B surface antibody [20].

Like hepatitis B, chronic hepatitis C infection has the similar sequelae of cirrhosis and hepatocellular carcinoma, and up to three quarters of infected persons are unaware of their infection status [22]. Testing for hepatitis $\mathrm{C}$ among donors requires serologic EIA testing for the hepatitis $\mathrm{C}$ antibody and NAT for viral particles [8].

\section{Syphilis}

Serologic testing for syphilis is recommended by the FDA for screening and diagnosis because the bacterial source, Treponema pallidum, cannot be cultured [8, 23]. Serologic testing of donors initially involves the nontreponemal assays, such as rapid plasma regain (RPR) and venereal disease research laboratory (VDRL) [8]. These assays detect antibodies for phospholipids which are present not only on T. pallidum but also occur in autoimmune or inflammatory conditions [24]. Positive nontreponemal assays should be confirmed 
with FDA-approved treponemal-based assays, such as fluorescent treponemal antibody (FTA), $T$. pallidum particle agglutination (TP-PA), or EIA for specific antibodies to T. pallidum [8]. Unlike nontreponemal assays, treponemal-based assays remain positive for years after treatment and infection [25]. Donors may be eligible for oocyte donation if the treponemal-based assays are negative.

\section{Neisseria Gonorrhoeae and Chlamydia Trachomatis}

Testing for these two sexually transmitted infections involves cervical cultures or nucleic acid amplification tests [8]. Samples may be taken from urine or a swab from the cervix, urethral meatus, or vagina [8]. As discussed previously, egg donors with a history of these infections should generally be discouraged from donation.

\section{Blood Type}

Testing potential donors for blood type and Rh factor is not considered mandatory by the FDA or ASRM. It may be recommended to ensure compatibility with the maternal genotype. The potential for $\mathrm{Rh}$ incompatibility and the obstetric implications (e.g., hemolytic disease of the fetus, hydrops fetalis, and intrauterine fetal demise) should be divulged to recipients.

\section{Genetics}

The minimum genetic screening for oocyte donors involves ruling out any history of Mendelian disorders, such as autosomal dominant disorders, $\mathrm{X}$-linked disorders, and autosomal recessive inheritance. If donors are from certain ethnic backgrounds that are high risk for genetic disorders, they should be tested for their carrier status. For example, patients of African or Mediterranean descent should have cell blood counts and hemoglobin electrophoresis to assess any risk for sickle cell anemia or beta-thalassemia among future offspring [26]. Those individuals with Southeast Asia ancestry are at high risk for alpha-thalassemia and should be screened with DNA-based testing as they may have normal hemoglobin electrophoreses [27, 28]. Donors of Askenazi jewish descent should be screened for Tay-Sachs disease, Gaucher disease, Fragile X syndrome, Fanconi anemia, Canavan disease, Niemann-Pick type A, Bloom syndrome, maple syrup disease, glycogen storage syndromes, familial dysautonomia, and mucolipidosis type IV [17, 29].

We believe that all ethnicities should be screened for cystic fibrosis and spinal muscular atrophy (SMA) due to its relatively high carrier rate prevalence. The carrier frequency of cystic fibrosis among the Caucasian, Hispanic, and African American populations is 1 in 25, 1 in 46, and 1 in 65, respectively [30]. The carrier risk of SMA is 1 in 47 among the Caucasian population and 1 in 67 among the Ashkenazi Jewish population [31]. Among the Asian, African American, and Hispanic populations, the carrier risk is 1 in 59, 1 in 72, and 1 in 68, respectively [31].

Donors heterozygous for autosomal recessive disorders need not always be excluded, but screening requires testing of the carrier status of the recipient's partner. It is also important to ascertain a history of major malformations of multifactorial or polygenic etiology that are associated with any serious functional or cosmetic handicap (e.g., cardiac and uterine malformations). Donors are excluded if they have any known karyotypic abnormality or any significant familial disease with a major genetic component among first-degree relatives (e.g., BRCA-positive breast cancer) [8].

Routine karyotyping and testing for TaySachs or Fragile $\mathrm{X}$ is not currently recommended by ASRM for donor genetic screening but may also be considered. A recent retrospective study compared the ASRM screening guidelines with enhanced universal screening, which included Tay-Sachs, Fragile X, and karyotype analysis [32]. Over a 12-year period, investigators found an additional 25 candidate exclusions with enhanced universal screening of 1,300 potential donors, making up $19 \%$ of all genetic exclusions [32]. Although karyotyping is not customarily recommended in the USA, it is part of donor screening in some European countries. An IVF center in Valencia, Spain, which regularly analyzes the karyotypes potential donors, found that $1.4 \%$ of 
karyotypes were abnormal over a 10 -year period [33]. At this time, enhanced genetic screening does not fall under current ASRM guidelines; however, Counsyl Medical Genomics offers a comprehensive panel of more than 100 recessive single gene disorders that would broadly expand surveillance (Table 4.5) [34]. However, until there is more research into the cost-effectiveness of testing and how best to advise patients of the many results, it cannot be routinely recommended.

\section{Ovarian Reserve}

Restricting the age of potential donors is important in maintaining a successful program, and retrieving a good number of oocytes is central to successful pregnancy outcomes. Selecting an optimal donor and predicting ovarian response based solely upon age are limited by individual variation in response to ovarian stimulation. In conjunction with age, ovarian reserve testing among prospective donors is useful in assessing the donor's ovarian reserve status, predicting ovarian response and assessing risk for OHSS.

Anti-Müllerian hormone (AMH) represents an accurate marker of ovarian response to gonadotropin stimulation in IVF cycles. Several studies have suggested that AMH accurately predicts poor ovarian response $[35,36]$. Our center found significant correlations between AMH and the number of oocytes retrieved and estradiol levels among oocyte donors (Table 4.6) [37]. AMH may be useful in predicting IVF cycle outcomes and helpful in individualizing dosing protocols [37]; however, further studies are needed.

Investigators have also suggested that antralfollicle count (AFC) could be used similarly to predict oocyte donor response to controlled ovarian hyperstimulation [38]. Follicle-stimulating hormone (FSH) in conjunction with estradiol has been used for assessment of ovarian reserve; however, they have not been found to reliably predict ovarian reserve in young patients [39]. Premature diminished ovarian reserve would be important to ascertain early in the donor screening process not only for the effect on oocyte yield but also for the knowledge of the donor.
The use of CGG triple nucleotide repeats on the Fragile $\mathrm{X}(F M R l)$ gene has also been suggested as an addition to future ovarian reserve testing among oocyte donors given its association with premature ovarian failure [40]. One pilot study suggested that FMR1 gene testing in conjunction with age-specific AMH may be a useful adjunct measure [41].

\section{Cervical Dysplasia}

Prospective donors should undergo screening for cervical dysplasia in accordance to current recommendations by the American Society for Colposcopy and Cervical Pathology (ASSCP). At presentation to our center, donors should have record of a normal PAP smear within the past 3 years. Abnormal cytology or pathology should be referred for appropriate treatment according to current guidelines prior to participating.

\section{Toxicology}

Information regarding current and past drug and/or alcohol abuse can be obtained during the donor evaluation of past medical and psychosocial history; therefore, many programs do not routinely perform urine drug screening of donors. Over a 4-year period of universal drug screening, one center found positive urine toxicology in $7 \%$ of their donor population who initially denied current drug use [42]. Though toxicology testing is not routinely performed, centers may wish to consider testing potential donors with a worrisome past medical or psychosocial history. We routinely test all of our donors, and from 2004 to 2010, we found $3 \%$ of women screened to have positive urine toxicology [43]. Our center uses the Quest Diagnostics ten drug urine toxicology panel (Table 4.7).

\section{Psychological Screening}

ASRM currently recommends psychological and social assessment of oocyte donors by a qualified mental health professional [8]. Typically, these 


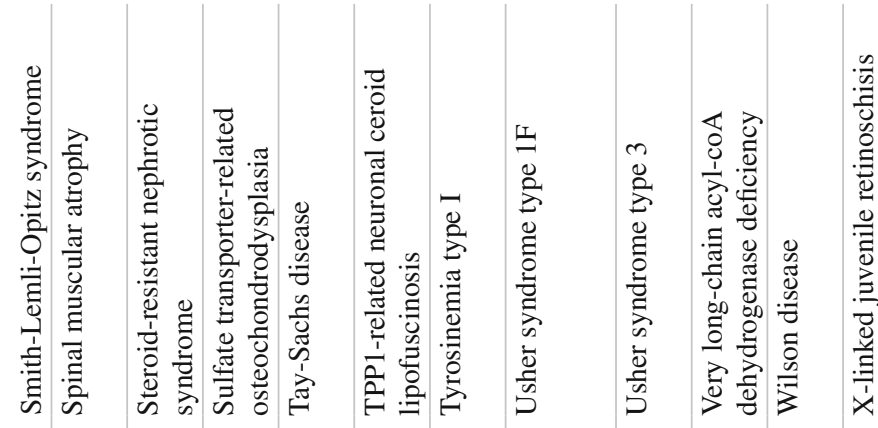

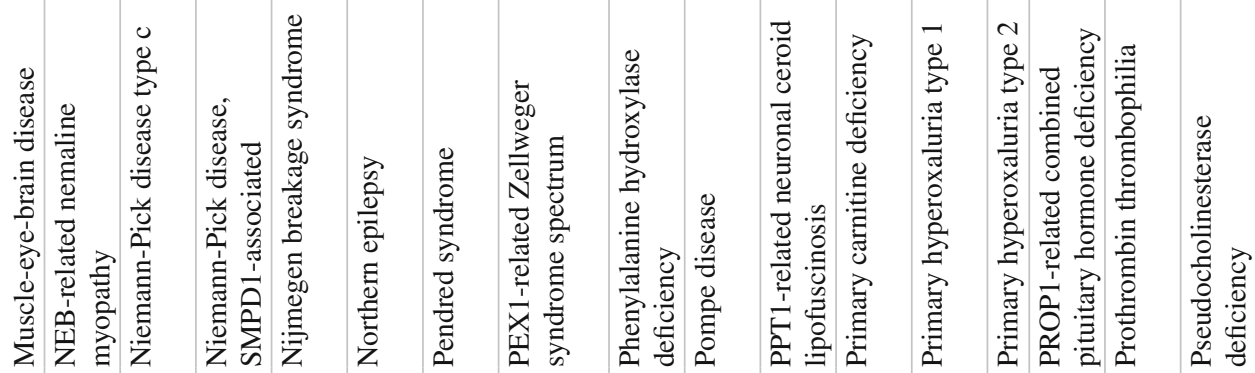

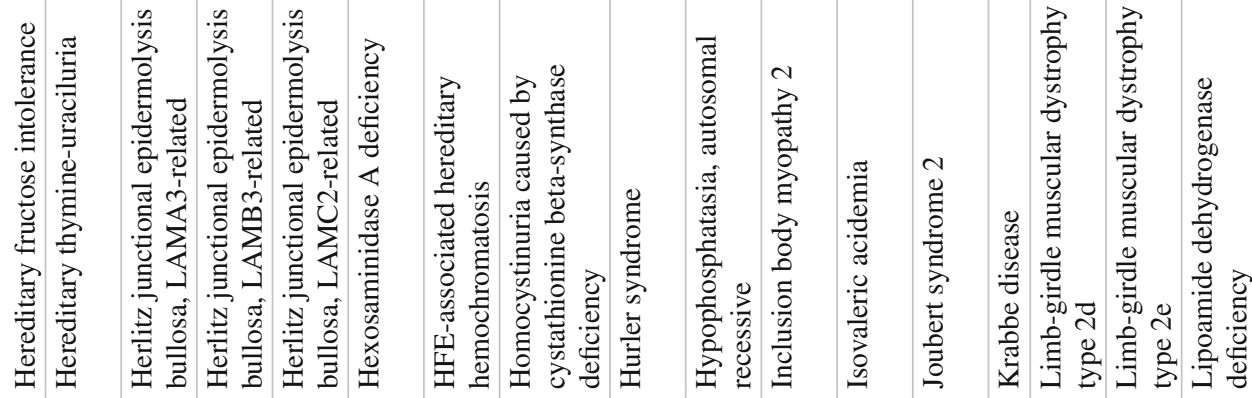




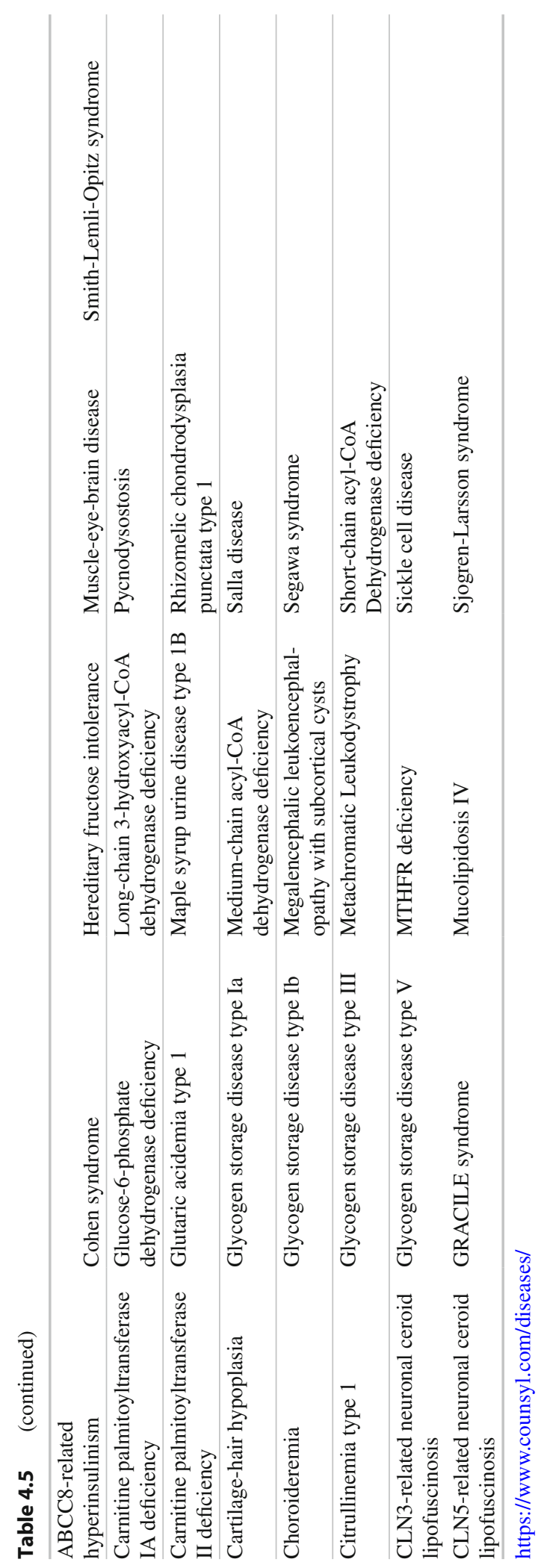


Table 4.6 Association of AMH to donor and outcome parameters [34]

\begin{tabular}{llllll}
\hline & Donor age & Donor BMI & Peak estradiol & Total oocytes & Total gonadotropin dose \\
\hline$r$ (Pearson) & -0.042 & -0.158 & 0.235 & 0.232 & -0.35 \\
\hline$P$-value & NS & NS & 0.024 & 0.024 & 0.05 \\
\hline
\end{tabular}

$B M I$ body mass index, NS not statistically significant

Table 4.7 Quest diagnostics urine toxicology screen

Amphetamines
Benzodiazepines
THC (marijuana)
Cocaine
Methadone
Methaqualone (Quaaludes)
Opiates
Phencyclidine (PCP/angel dust)
Barbiturates
Propoxyphene (Darvon)
EMIT enzyme multiplied immunoassay technique

are psychologists, psychiatrists, or social workers familiar with issues pertaining to gamete donation. The medical health professional collects a thorough psychosocial history that includes family history, educational background, assessment of emotional stability, motivation to donate, current life stressors and coping skills, difficult or traumatic reproductive history, interpersonal relationships, sexual history, travel history, history of major psychiatric and personality disorders, substance abuse in donor or first-degree relatives, legal history, and history abuse or neglect [8]. Presence of significant psychopathology, positive family history of heritable psychiatric disorders, substance abuse, two or more first-degree relatives with substance abuse, current use of psychoactive medications, history of sexual or physical abuse without professional treatment, excessive stress, marital instability, impaired cognitive functioning, mental incompetence, and high-risk sexual practices may warrant exclusion of the prospective donor [8]. Ineligible donors should be explained the reasons for their exclusion with appropriate referral as needed [8].

In the case of a known donor, complete psychosocial evaluation and counseling is strongly recommended for both the donor and the recipients in order to fully assess the potential impact of donation, pregnancy, and even treatment failure on their future relationship. Evaluation needs to rule out any undue financial or emotional coercion or enticement [8]. Programs should confirm that the donor maintains autonomy in her decision to participate and understands the potential effects on her relationships with the recipients and other family members if she withdraws or continues to participate [44].

The details of the psychological evaluation are not specifically defined and vary between centers. As a result the Mental Health Professional Group created guidelines for psychological testing of prospective oocyte donors. The Minnesota Multiphasic Personality Inventory (MMPI) has been traditionally used to evaluate oocyte donors in order to differentiate healthy individuals from those predisposed to psychiatric disorders [45]. The second edition of this test, MMPI-2, has been shown to distinguish between those individuals who answer truthfully and those who try to underplay any psychopathologic behavior. Prior studies have found differences in MMPI-2 scores between donors who completed a donation cycle and those who were psychologically excluded, but were not able to reliably differentiate those accepted who would subsequently be noncompliant [46, 47]. ASRM guidelines do not currently require psychological testing with MMPI-2, but centers often use this evaluation in addition to their current psychological screening methods to assess potential oocyte donors.

\section{Summary}

Screening and selection of egg donors is a comprehensive process in order to help protect the safety and health of donors, recipients, and future offspring. For potential donors, it is a stepwise 
sequence of events beginning with the review possible risks associated with treatment. Once an egg donor consents, after understanding the risks and their rights, duties, and obligations, the process of donor screening and selection belongs to the egg donor program under the guidance and recommendations of the FDA, ASRM, and local state health departments. Obtaining a thorough medical history, performing a complete physical exam, infectious disease testing, genetic screening, and psychosocial evaluation require a dedicated, organized, and thorough multidisciplinary team of reproductive endocrinologists, nurses, mental health professionals, and social workers. As further research develops regarding the screening and selection process, there will likely be further changes of the guidelines discussed in this chapter, and it will be prudent to have a multidisciplinary team to help make adjustments to these changes an ongoing and efficient process. For now, following the current recommendations and guidelines will assist programs in the appropriate selection of oocyte donors and maximize the chances for a successful pregnancy in recipients.

\section{Editor's Commentary}

The participation of young healthy women as egg donors has engendered more controversy and public scrutiny than perhaps any other aspect of egg donation. It has been attacked from the beginning by critics of the method as a dangerous, exploitive, unprofessional, and sexist practice, and yet it endures. To combat such ferocious criticism, it is imperative that practitioners of egg donation pay careful attention to every aspect of donor recruitment and participation. Responsible practice fosters good outcomes and continued success. There will always be a great amount of pressure to either supply donors or accept donors that may be less than ideal (e.g., PCOS, hypothalamic amenorrhea), but doctors need and must be able to say no when faced with choices that pose undue risk to all participating.
For the first decade of practice, the model for screening donors was essentially lifted from the manner in which men were screened prior to donating sperm. However, sperm and egg donation shares little in common with respect to time involvement and risk of participation, and therefore the need for more specific and detailed professional guidelines and safety measures was necessary to protect the women donating eggs, the women receiving them, and most importantly the child that results from their collaborative efforts. Dr. Thornton reviews the important basic requirements for establishing an egg donor program. Many of these professional activities are now being provided by "agencies," which are typically not run by physicians, who then supply patients or programs with "matches." Regardless of whether an agency supplies the donor or a program chooses to screen potential donors themselves, the outlined steps provided in this chapter are crucial to follow in order to ensure safety and health. Ultimately, the responsibility falls to the doctors providing the hands-on care and in every case full knowledge of the donor's pedigree, health history, and reason for participating must be known.

During the developmental years of the method, egg and embryo donors were married middle-class mothers in their early 30 s. Today, we are working with the youngest women in the history of the technique, typically in their midtwenties, single, and without children. Injury to any one of them is catastrophic and ironically may in fact leave them infertile. We also can assume that some of these women will later experience infertility themselves and naturally will assume that their work as an egg donor contributed to their problem. Disclosure of all potential risks, including later regret, is difficult to do without frightening potential donors away. However, I believe it is better to inform each of them of the known complications and lose a few candidates rather than to perform 
a procedure that later leads to lifelong regret. It has always been my hope that every egg donor will look back on the experience with pride and satisfaction for having provided a truly unique gift of life.

\section{References}

1. Morris RS, Sauer MV. New advances in the treatment of infertility in women with ovarian failure. Curr Opin Obstet Gynecol. 1993;5(3):368-77.

2. Sauer MV, Paulson RJ, Macaso TM, Francis-Hernandez M, Lobo RA. Establishment of a nonanonymous donor oocyte program: preliminary experience at the University of Southern California. Fertil Steril. 1989;52:433-6.

3. Noggle S, Fung HL, Gore A, et al. Human oocytes reprogram somatic cells to a pluripotent state. Nature. 2011;478(7367):70-5.

4. Technology SfAR. Clinical summary report: all SART member clinics. Available at: https://www.sartcorsonline.com/. Birmingham (AL): SART; 2009; Retrieved 25 Sept 2011.

5. Gunning J. Oocyte donation: the legislative framework in Western Europe. Hum Reprod. 1998;13 Suppl 2:98-102; discussion 103-4.

6. Covington SN, Gibbons WE, Society for Assisted Reproductive Technology. What is happening to the price of eggs? Fertil Steril. 2007;87(5):1001-4.

7. Luk J, Petrozza JC. Evaluation of compliance and range of fees among American Society for Reproductive Medicine-listed egg donor and surrogacy agencies. J Reprod Med. 2008;53(11):847-52.

8. Practice Committee of American Society for Reproductive Medicine, Practice Committee of Society for Assisted Reproductive Technology. 2008 guidelines for gamete and embryo donation: a practice committee report. Fertil Steril. 2008;90(5 Suppl):S30-44.

9. US Food and Drug Administration (FDA). Guidance for industry: eligibility determination for donors of human cells, tissues, and cellular and tissue-based products. US Department of Health and Human Services. 2008.

10. Battaglia DE, Goodwin P, Klein NA, Soules MR. Influence of maternal age on meiotic spindle assembly in oocytes from naturally cycling women. Hum Reprod. 1996;11(10):2217-22.

11. Pellestor F, Andreo B, Arnal F, Humeau C, Demaille J. Maternal aging and chromosomal abnormalities: new data drawn from in vitro unfertilized human oocytes. Hum Genet. 2003;112(2):195-203.

12. Munne S, Alikani M, Tomkin G, Grifo J, Cohen J. Embryo morphology, developmental rates, and maternal age are correlated with chromosome abnormalities. Fertil Steril. 1995;64(2):382-91.
13. Ethics Committee of the American Society for Reproductive M. Interests, obligations, and rights of the donor in gamete donation. Fertil Steril. 2009;91(1):22-7.

14. Schaefer GO, Sinaii N, Grady C. Informing egg donors of the potential for embryonic research: a survey of consent forms from U.S. in vitro fertilization clinics. Fertil Steril. 2012;97(2):427-33.

15. Sauer MV. Defining the incidence of serious complications experienced by oocyte donors: a review of 1000 cases. Am J Obstet Gynecol. 2001;184(3):277-8.

16. Practice Committee of American Society for Reproductive M. Repetitive oocyte donation. Fertil Steril. 2008;90(5 Suppl):S194-5.

17. Sauer MV, Kavic SM. Oocyte and embryo donation 2006: reviewing two decades of innovation and controversy. Reprod Biomed Online. 2006;12(2):153-62.

18. Venn A, Watson L, Lumley J, Giles G, King C, Healy D. Breast and ovarian cancer incidence after infertility and in vitro fertilisation. Lancet. 1995;346(8981): 995-1000.

19. Fiebig EW, Wright DJ, Rawal BD, et al. Dynamics of HIV viremia and antibody seroconversion in plasma donors: implications for diagnosis and staging of primary HIV infection. AIDS. 2003;17(13):1871-9.

20. Mast EE, Weinbaum CM, Fiore AE, et al. A comprehensive immunization strategy to eliminate transmission of hepatitis B virus infection in the United States: recommendations of the Advisory Committee on Immunization Practices (ACIP) part II: immunization of adults. MMWR Recomm Rep. 2006;55(RR-16): 1-33; quiz CE31-4.

21. Liaw YF, Chu CM. Hepatitis B virus infection. Lancet. 2009;373(9663):582-92.

22. Hagan H, Campbell J, Thiede H, et al. Self-reported hepatitis $\mathrm{C}$ virus antibody status and risk behavior in young injectors. Public Health Rep. 2006;121(6): 710-9.

23. Fieldsteel AH, Cox DL, Moeckli RA. Cultivation of virulent Treponema pallidum in tissue culture. Infect Immun. 1981;32:908-15.

24. Larsen SA, Steiner BM, Rudolph AH. Laboratory diagnosis and interpretation of tests for syphilis. Clin Microbiol Rev. 1995;8(1):1-21.

25. Young H, Pryde J, Duncan L, Dave J. The architect syphilis assay for antibodies to Treponema pallidum: an automated screening assay with high sensitivity in primary syphilis. Sex Transm Infect. 2009;85(1):19-23.

26. Lorey FW, Arnopp J, Cunningham GC. Distribution of hemoglobinopathy variants by ethnicity in a multiethnic state. Genet Epidemiol. 1996;13(5):501-12.

27. Tang HS, Zhou JY, Xie XM, Li R, Liao C, Li DZ. Screening for common nondeletional alpha-thalassemias in Chinese newborns by determination of $\mathrm{Hb}$ Bart's using the Sebia Capillarys 2 electrophoresis system. Hemoglobin. 2012;36(2):196-9.

28. Li R, Liao C, Li D, Li J. High-resolution melting analysis of the three common nondeletional alpha-thalassemia mutations in the Chinese population: Hbs Constant Spring, Quong Sze and Westmead. Hemoglobin. 2010;34(6):587-93. 
29. ACOG Committee on Genetics. ACOG committee opinion No. 442: preconception and prenatal carrier screening for genetic diseases in individuals of eastern European Jewish descent. Obstet Gynecol. 2009;114(4):950-3.

30. Richards CS, Bradley LA, Amos J, et al. Standards and guidelines for CFTR mutation testing. Genet Med. 2002;4(5):379-91.

31. Sugarman EA, Nagan N, Zhu H, et al. Pan-ethnic carrier screening and prenatal diagnosis for spinal muscular atrophy: clinical laboratory analysis of $>72,400$ specimens. Eur J Hum Genet. 2012;20(1):27-32.

32. Reh A, Amarosa A, Licciardi F, Krey L, Berkeley AS, Kump L. Evaluating the necessity for universal screening of prospective oocyte donors using enhanced genetic and psychological testing. Hum Reprod. 2010;25(9):2298-304.

33. Garrido N, Zuzuarregui JL, Meseguer M, Simon C, Remohi J, Pellicer A. Sperm and oocyte donor selection and management: experience of a 10 year follow-up of more than 2100 candidates. Hum Reprod. 2002; 17(12):3142-8.

34. Srinivasan BS, Evans EA, Flannick J, et al. A universal carrier test for the long tail of Mendelian disease. Reprod Biomed Online. 2010;21(4):537-51.

35. Seifer DB, MacLaughlin DT, Christian BP, Feng B, Shelden RM. Early follicular serum mullerian-inhibiting substance levels are associated with ovarian response during assisted reproductive technology cycles. Fertil Steril. 2002;77(3):468-71.

36. Nakhuda GS, Sauer MV, Wang JG, Ferin M, Lobo RA. Mullerian inhibiting substance is an accurate marker of ovarian response in women of advanced reproductive age undergoing IVF. Reprod Biomed Online. 2007;14(4):450-4.

37. Nakhuda GS, Douglas NC, Thornton MH, Guarnaccia MM, Lobo R, Sauer MV. Anti-Mullerian hormone testing is useful for individualization of stimulation protocols in oocyte donors. Reprod Biomed Online. 2011;22 Suppl 1:S88-93.
38. Barreto Melo MA, Garrido N, Alvarez C, et al. Antral follicle count (AFC) can be used in the prediction of ovarian response but cannot predict the oocyte/ embryo quality or the in vitro fertilization outcome in an egg donation program. Fertil Steril. 2009;91(1): 148-56.

39. Esposito MA, Coutifaris C, Barnhart KT. A moderately elevated day 3 FSH concentration has limited predictive value, especially in younger women. Hum Reprod. 2002;17(1):118-23.

40. Gleicher N, Weghofer A, Oktay K, Barad D. Relevance of triple CGG repeats in the FMR1 gene to ovarian reserve. Reprod Biomed Online. 2009;19(3):385-90.

41. Gleicher N, Weghofer A, Barad DH. Can egg donor selection be improved? A pilot study. Reprod Biol Endocrinol. 2010;8:76.

42. Westphal L, Bendikson K. Evaluation of urine toxicology screens in an oocyte donor population. J Assist Reprod Genet. 2005;22(2):103-4.

43. Levine BA, Klein J, Charles A, Sauer MS. Routine toxicology screening of oocyte donors. Presented at the 67 th annual meeting of the American Society for Reproductive Medicine, Orlando. 2011.

44. American Society for Reproductive Medicine Ethics Committee. Family members as gamete donors and surrogates. Fertil Steril. 2003;80(5):1124-30.

45. Lessor R, Cervantes N, O’Connor N, Balmaceda J, Asch RH. An analysis of social and psychological characteristics of women volunteering to become oocyte donors. Fertil Steril. 1993;59(1):65-71.

46. Klock SC, Stout JE, Davidson M. Analysis of Minnesota multiphasic personality inventory-2 profiles of prospective anonymous oocyte donors in relation to the outcome of the donor selection process. Fertil Steril. 1999;72(6):1066-72.

47. Klock SC, Covington SN. Minnesota Multiphasic Personality Inventory (MMPI-2) profiles in the assessment of ovum donors. Fertil Steril. 2010;94(5): 1684-8. 